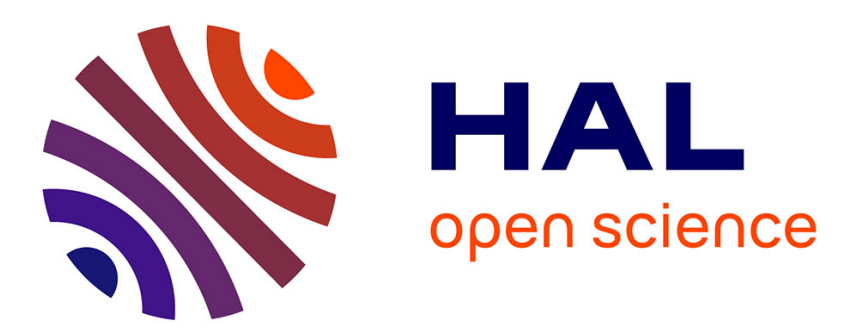

\title{
Wave propagation through dense vertical cylinder arrays: 3D experimental study
}

G. Arnaud, Vincent Rey, Julien Touboul, Damien Sous

\section{To cite this version:}

G. Arnaud, Vincent Rey, Julien Touboul, Damien Sous. Wave propagation through dense vertical cylinder arrays: 3D experimental study. International Journal of Ocean and Coastal Engineering, inPress. hal-02127506

\section{HAL Id: hal-02127506 \\ https://hal.science/hal-02127506}

Submitted on 13 May 2019

HAL is a multi-disciplinary open access archive for the deposit and dissemination of scientific research documents, whether they are published or not. The documents may come from teaching and research institutions in France or abroad, or from public or private research centers.
L'archive ouverte pluridisciplinaire HAL, est destinée au dépôt et à la diffusion de documents scientifiques de niveau recherche, publiés ou non, émanant des établissements d'enseignement et de recherche français ou étrangers, des laboratoires publics ou privés. 


\title{
Wave propagation through dense vertical cylinder arrays: 3D experimental study
}

\author{
Gwendoline Arnaud \\ Université de Toulon, Aix-Marseille Université, CNRS/INSU, IRD, MIO UM110, Mediterranean \\ Institute of Oceanography, La Garde, France \\ gwendoline.arnaud@univ-tln.fr \\ Vincent Rey \\ Université de Toulon, Aix-Marseille Université, CNRS/INSU, IRD, MIO UM110, Mediterranean \\ Institute of Oceanography, La Garde, France \\ vincent.rey@univ-tln.fr \\ Julien Touboul \\ Université de Toulon, Aix-Marseille Université, CNRS/INSU, IRD, MIO UM110, Mediterranean \\ Institute of Oceanography, La Garde, France \\ julien.touboul@univ-tln.fr \\ Damien Sous \\ Université de Toulon, Aix Marseille Université, CNRS/INSU, IRD, MIO UM110, Mediterranean \\ Institute of Oceanography (MIO), La Garde, France \\ Univ. Pau \& Pays Adour / E2S UPPA, Laboratoire des Sciences de l'Ingénieur Appliquées à la \\ Mécanique et au Génie Electrique (SIAME) - MIRA, EA4581, 64600, Anglet, France \\ damien.sous@mio.osupytheas.fr
}

\begin{abstract}
The purpose of this research work is to study the diffraction of surface gravity waves propagating through rectangular porous medium in three dimensions. The considered porous structure consists in dense arrays of surface piercing vertical cylinders. Experiments for different regular wave conditions have been carried out, especially for three wave frequencies. The experimental data of wave refraction-diffraction and reflection have been compared to computed results from potential linear theory solved with an integral matching method. Comparison with a previous 2D study about wave propagation through porous medium in a $10 \mathrm{~m}$ long wave flume is also discussed in order to highlight the refraction-diffraction effect due to the porous structure.
\end{abstract}

Keywords: Wave;Porous medium;Dissipation;Diffraction;Reflection; Integral matching numerical method

\section{Introduction}

Coastal countries are generally facing the over-urbanization of their coastlines and have to consider the increasing risks of damages in the nearshore due to climate 
change. Engineers have proposed many solutions to shelter the shore, more and more designed to maintain the environmental quality of coastal waters.

A classical option is to install detached offshore breakwaters to scatter the waves. Wave energy is then strongly reduced at the shore, due to both dissipation on the rocky structure and redistribution of the wave field along the coast. Series of breakwaters along the coast are often used, their efficiency being dependent on both their width and spacing (Dalrymple and Martin, 1990; Abul-Azm and Williams, 1997). These breakwaters being displayed parallel to the shore, water circulation is then maintained behind them.

Porous structures are also among the proposed solutions, for their ability to dissipate part of the incoming wave energy and to maintain the water circulation. Water wave still propagates through the structure with a significant damping. However, modelling such structures remains extremely difficult, since the behaviour of water waves within such complex, inhomogeneous media remains partially misunderstood. Still, a few attempts can be found within the literature. For instance, Sollitt and Cross (1972) have considered a complex expression of the dispersion equation for water waves propagating within the porous media, for which the complex wavenumbers include a propagating term and a dissipative term. The case of emerging porous media including superimposed structures have been studied by $\mathrm{Yu}$ and Chwang (1994). In their work, evanescent modes were taken into account since the porosity was not constant along the vertical boundaries extending from the bottom to the free surface.

Interesting insight on the wave propagation through porous media can also be gained from hydrodynamics of dense arrays of vertical cylinders often used to model wetland vegetation or coral reefs (Lowe, 2005a,b; Maza et al., 2015). In these studies, both the size and cylinders' spacing are small compared to the wave wavelength. A general trend is the significant wave damping when propagating through the cylinder array (Mei et al. (2014) and references cited herein). Generally, the wave propagation through arrays of cylinders are generally modeled by three kinds of mathematical models. The flow can be directly 3D numerically solved, with a full discretization of the domain (Ma et al., 2013). If this method allows a complete description of the flow, it requires a strong computing power. To overcome this time consuming approach, several authors have investigated a homogenization theory which associates micro- and macro-scales perturbation techniques compared to the wavelength-scale of study (Liu et al., 2015; Yang et al., 2015; Chang et al., 2017a,b), this approach allows on one hand a fine description at the scale of the structures, and on the other hand their effect at the wave scale. In the present study, the porous medium is considered as a homogeneous structure at the wave scale since both the size and spacing of the cylinders remain small compared to the wavelength. This assumption allows to define an equivalent wavelength within the porous medium as described in the 2D case by Arnaud et al. (2017). Numerical resolution at the macroscale using boundary integral matching method between the different domains is then consid- 
ered. Arrays of vertically emerging bodies have also been widely studied in ocean engineering in the context of wave diffraction by cylinder arrays and wave loads on these structures (see e.g. Kagemoto and Yue (1986); Ohl et al. (2001) and references cited herein). However, these studies, which are often based on wave field expansion in a circular coordinate system, assume sparse arrays of cylinders. Application to either sparse or dense emerging vertical cylinder arrays was recently proposed by Rey et al. (2018) for uneven periodic cylinders normally to the incoming wave direction. For dense arrays of vertical emerging cylinders, both cylinder diameter and spacing are small compared to the wavelength. With this approximation, the cylinder array can be considered as a porous medium, as done by Arnaud et al. (2017) in a one-dimensional case.

The purpose of the present work is to improve the understanding of wave propagation through a porous structure involving a cylinder array homogeneous at the wave scale, in terms of coastal protection. To do so, the general expressions of velocity potentials are expanded in a cartesian frame of reference for each finite or semi-finite medium composing the wave propagation media. The present analytical model relies on the complex dispersion relation presented by Yu and Chwang (1994) for the wavelength determination within the porous medium. Since the porous media is considered as homogeneous at the wave scale a linear damping approach is used to take into account wave dissipation inside the porous media. Wave damping parametrization takes advantage of the careful experiments including various wave steepnesses and periods carried out in the 2D case by Arnaud et al. (2017) for similar structure.. The problem solution is obtained by integral matching method resolving continuity equations of velocity and pressure at vertical boundaries between domains. The model is confronted to a corresponding physical model, and the performance of the system is interpreted by intercomparing this model with experimental results.

\section{Experimental setup}

\subsection{Wave basin}

Experiments have been carried out in a basin $10 \mathrm{~m}$ long and 2.60 width, of the Engineering School of the University of Toulon (SeaTech). At one end, a "corner" type wave-maker is used to generate regular waves in the range $0.7-2.5 \mathrm{~Hz}$. At the other end, a parabolic porous beach is installed to maximize incoming waves dissipation and avoid spurious reflection. The typical water depth is one meter but in the present study an additional bottom was used in order to fulfill finite water depth conditions. The bottom is then made of a sloping ramp from the wavemaker and the horizontal part supporting the porous medium and imposing a constant water depth $h=0.23 \mathrm{~m}$. 


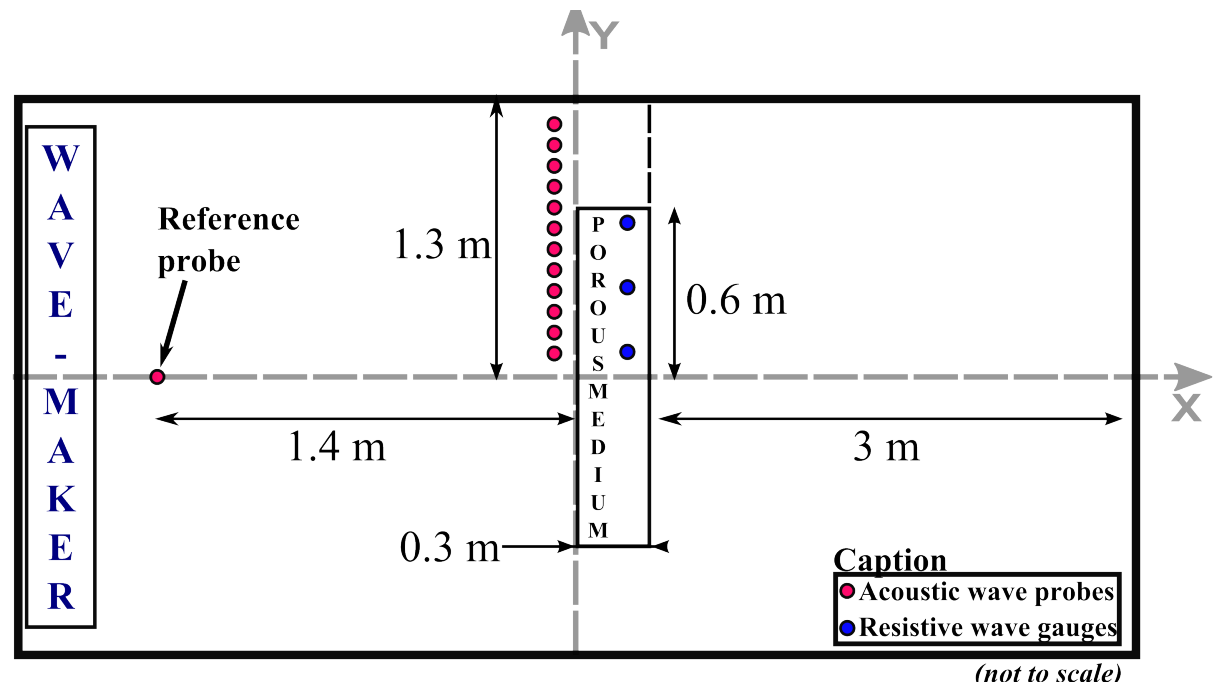

Figure 1. Experimental set-up

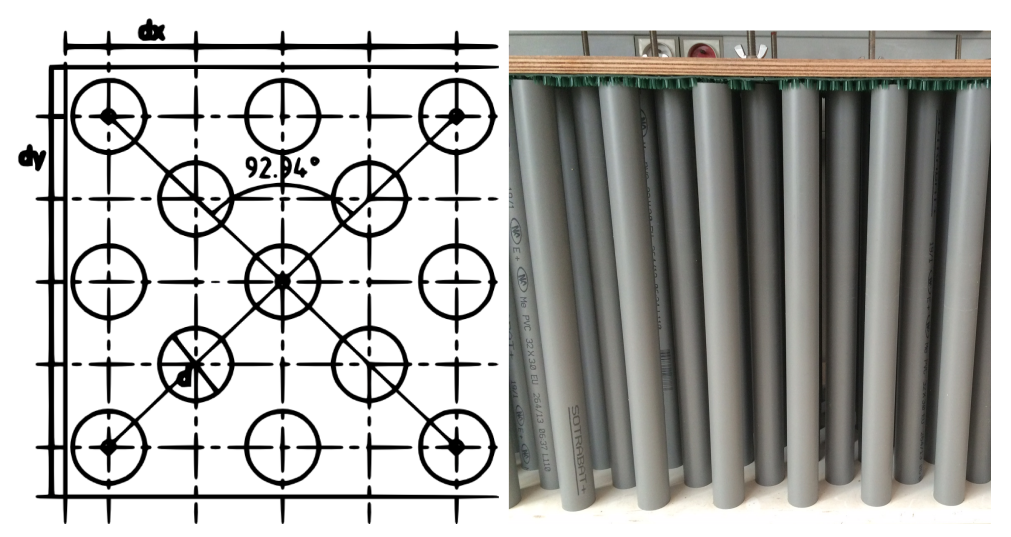

Figure 2. Scheme of top view and photography of side view of porous medium

\subsection{Porous medium}

The model porous medium is made of a vertical cylinder array. Cylinders are uniformly staggered along two perpendicular axes. These axes form a $45^{\circ}$ angle with 
the longitudinal axis of the structure. A detailed study of such structure is presented in Arnaud et al. (2017) in the 2D case. In the present study, the cylinder diameter is $D=0.032 \mathrm{~m}$. The length of the structure is $L=0.30 \mathrm{~m}$ and the width is $2 d_{p}=1.20$ $\mathrm{m}$. The distances between cylinders along horizontal axis are $d x=0.0384 \mathrm{~m}$ and $d y=0.0365 \mathrm{~m}$, respectively (see Fig. 2). The porosity $\gamma$ and the specific surface $s$ are defined in Eqs. 1 and 2, respectively.

$$
\begin{gathered}
\gamma=\frac{2 d_{p} L-N \pi(D / 2)^{2}}{2 d_{p} L} \\
s=\frac{\pi D N}{2 d_{p} L}
\end{gathered}
$$

where $N$ is the number of cylinders of porous medium, here $N=120$.

In the present study $\gamma=0.7$ and $s=33 \mathrm{~m}^{-1}$.

\subsection{Sensors and experimental conditions}

The free surface has been measured with thirteen acoustic wave probes deployed in the near vicinity of the porous structure (see Fig. 1). The sampling frequency is $40 \mathrm{~Hz}$. Measurements of the wave amplitude are carried out around the structure according to a grid of $0.10 \times 0.10 \mathrm{~m}^{2}$ by moving the racks of gauges.

For this experiment, wave frequency and wave height are chosen in intermediate to almost deep water conditions. Frequencies and amplitudes corresponding to the dimensionless wavelength inside and around the porous medium are given in Table 1. Note that the conditions studied concern non breaking waves. $\lambda$ and $\lambda_{p}$ are the

Table 1. Wave conditions

\begin{tabular}{|l|ccc|cc|}
\hline Frequency & \multicolumn{3}{|c|}{ Amplitudes $(\mathrm{m})$} & $h / \lambda$ & $h / \lambda_{p}$ \\
$f=1 \mathrm{~Hz}$ & 0.010 & 0.015 & 0.020 & 0.18 & 0.19 \\
$f=1.5 \mathrm{~Hz}$ & 0.010 & 0.015 & 0.020 & 0.34 & 0.38 \\
$f=1.8 \mathrm{~Hz}$ & 0.010 & 0.015 & & 0.48 & 0.53 \\
\hline
\end{tabular}

wave wavelengths outside and inside the porous structure, respectively.

\section{Theoretical Models}

The case of an incident wave in the $O x$ direction is considered. The wave propagates towards positive $x$ direction in the longitudinal axis of the basin. $O y$ is orthogonal to the incident wave direction with $y=0$ corresponding to the median axis of the basin. $O z$ is vertical upwards with $z=0$ corresponding to the position of the still water level. The coordinate system $(O, \vec{x}, \vec{y}, \vec{z})$ is a direct orthonormal coordinate system. Using symmetry properties with respect to $y=0$, the problem is solved for 

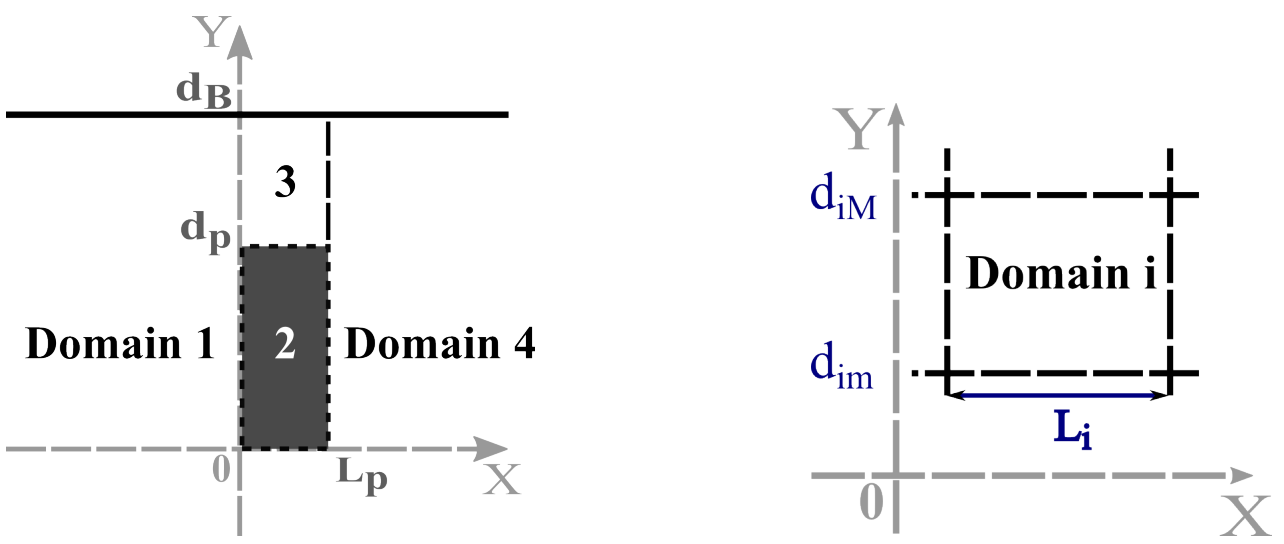

Figure 3. Scheme of half-width of tank with different domains considered in the theoretical model (left); Scheme of domain definition in the model (right)

$y \geq 0$. The problem solution is found writing a general expression of the potential in four domains:

- Domain 1: upstream, $0<y<d_{B}, x<0$,

- Domain 2: porous medium, $0<y<d_{p}, 0<x<L_{p}$,

- Domain 3: sidelong, $d_{p}<y<d_{B}, 0<x<L_{p}$,

- Domain 4: downstream, $0<y<d_{B}, x>L_{p}$,

and solving the continuity equations between adjacent domains (Fig. 3).

\subsection{General equations of velocity potentials}

For each rectangular domain $i$ of width $d_{i}, d_{m}<y<d_{M}$, and of length $L_{i}$, the general expression of the velocity potentials satisfying the impermeability conditions at $y=d_{i m}$ and $y=d_{i M}$ are of the form:

$$
\begin{aligned}
\Phi_{i}(x, y, z, t) & =\cosh \left[k_{i}(z+h)\right] \phi_{i}(x, y) e^{i \omega t} \\
& =\cosh \left[k_{i}(z+h)\right] \sum_{n=0}^{\infty}\left[A_{i, n}^{-} e^{-i k_{i x n} x}+A_{i, n}^{+} e^{+i k_{i x n} x}\right] \psi_{i, n}(y) e^{i \omega t}
\end{aligned}
$$

$i=1, \ldots, 4$, with

$$
\omega^{2} S_{r}=g k_{i} \tanh k_{i} h
$$

with $k_{i x n}=k_{i} \cos \theta_{n}$ and $k_{i y n}=k_{i} \sin \theta_{n}, n$ is a priori infinite (but practically truncated to an order $n=P$ ). For domains 1,3 and $4, S_{r}=1$ in contrast to domain 2 where $S_{r}=1+C_{m} \frac{1-\gamma}{\gamma}$ with $C_{m}=0.29$ (see Arnaud et al. (2017)). The wave number is $k_{i}$ in domains $i=1, \ldots 4$. In order to match the impermeability condition which assumes a zero normal velocity, at $y=d_{i m}$,

$$
\psi_{i, n}(y)=\cos \left[k_{i y n}\left(y-d_{i m}\right)\right]
$$


For an impermeable boundary at $y=d_{i M}, k_{i y n}$ must satisfy:

$$
k_{i y n}=\frac{n \pi}{d_{i M}-d_{i m}}
$$

The surface deformation $\eta_{i}(x, y, t)$ is given by:

$$
\frac{\partial \eta_{i}}{\partial t}=\left.\frac{\partial \Phi_{i}}{\partial z}\right|_{z=0}
$$

Wave amplitude is related to the potential amplitude through the following relation:

$$
A_{j}^{ \pm}=\frac{i \omega}{k_{j} \sinh k_{j} h} a_{j}^{ \pm}
$$

Since $\nabla^{2} \Phi_{i}=0$,

$$
k_{i x n}=\left(k_{i}^{2}-k_{i y n}^{2}\right)^{\frac{1}{2}}
$$

According to the $k_{x n}$ expression (eq. 9), from a given number of order $n_{p}>0, k_{x n}$ becomes pure imaginary. The direction of propagation $\theta_{n}$ for each mode $n, n \leq n_{p}$, with respect to the $O x$ direction of incident wave is given by:

$$
\theta_{i n}=\arctan \left[\frac{k_{i y n}}{k_{i x n}}\right]
$$

For $n>n_{p}$, the modes do not correspond anymore to propagative waves but to evanescent waves, and Eq. (9) can be written in the following form:

$$
k_{i x n}=i\left(\left|k_{i}^{2}-k_{i y n}^{2}\right|\right)^{\frac{1}{2}}
$$

For an impermeable boundary $y=d_{i m}$ and a permeable boundary $y=d_{i M}$, the function $\psi_{n}$ can still be written:

$$
\psi_{n}(y)=\cos \left[k_{i y n}\left(y-d_{i m}\right)\right]
$$

For a permeable boundary in $y=d_{i m}$ and an impermeable boundary $y=d_{i M}$, the function is written:

$$
\psi_{n}(y)=\cos \left[k_{i y n}\left(y-d_{i M}\right)\right]
$$

Expressions for domain $1\left(x<0,0<y<d_{B}\right)$ :

The incident wave is known. Writing that only the incident wave $(\theta=0)$ propagates toward $x>0$, and that evanescent modes do not diverge at $-\infty$, the general expression of the reduced potential $\phi_{1}(x, y)$ is the following:

$$
\phi_{1}(x, y)=A_{10}^{-} e^{-i k_{1 x 0} x} \psi_{10}(y)+\sum_{n=0}^{\infty} A_{1 n}^{+} e^{+i k_{1 x n} x} \psi_{1 n}(y)
$$


with

$$
\psi_{1 n}(y)=\cos \left[k_{1 y n}(y)\right] \text { and } k_{1 y n}=\frac{n \pi}{d_{B}}
$$

Expressions for domain $3\left(0<x<L_{p}, d_{p}<y<d_{B}\right)$ :

The general expression of reduced potential $\phi_{3}(x, y)$ is given by:

$$
\phi_{3}(x, y)=\sum_{n=1}^{\infty}\left[A_{3 n}^{-} e^{-i k_{3 x n} x}+A_{3 n}^{+} e^{+i k_{3 x n}\left(x-L_{p}\right)}\right] \psi_{3 n}(y)
$$

with

$$
\psi_{3 n}(y)=\cos \left[k_{3 y n}\left(y-d_{B}\right)\right]
$$

$k_{3 y n}$ is chosen of the form $k_{3 y n}=\frac{n \pi}{d_{B}}$, which corresponds to the decomposition mode in the absence of porous medium.

Expressions for domain $4\left(x>L_{p}, 0<y<d_{B}\right)$ :

Since there is no wave reflection from $+\infty$ whatever $\theta$ angle, and evanescent modes do not diverge at $+\infty$, the general expression of the reduced potential $\phi_{4}(x, y)$ is of the form:

$$
\phi_{4}(x, y)=\sum_{n=0}^{\infty} A_{4 n}^{-} e^{-i k_{4 x n}\left(x-L_{p}\right)} \psi_{4 n}(y)
$$

with

$$
\psi_{4 n}(y)=\cos \left[k_{4 y n}(y)\right] \text { and } k_{y 4 n}=\frac{n \pi}{d_{B}}
$$

Expressions for domain $2\left(0<x<L_{p}, 0<y<d_{B}\right)$ :

The general expression of the reduced potential $\phi_{2}(x, y)$ writes:

$$
\phi_{2}(x, y)=\sum_{n=0}^{\infty}\left[A_{2 n}^{-} e^{-i k_{2 x n} x}+A_{2 n}^{+} e^{+i k_{2 x n}(x-L)}\right] \psi_{2 n}(y)
$$

with

$$
\psi_{2 n}(y)=\cos \left[k_{2 y n}(y)\right]
$$

As for domain $3, k_{2 y n}$ is chosen of the form $k_{y 2 n}=\frac{n \pi}{d_{B}}$. In Arnaud et al. (2017) both linear and quadratic expression for wave damping were discussed. We consider here the damping is linear because the porous structure length $L_{p}$ is considered small compared to the wave's wavelength. A constant attenuation rate of wave amplitude is assumed in the direction of propagation within the porous medium dissipation. It is neglected elsewhere (domains 1, 3 and 4). For a partially stationary wave of 
the form $\eta_{2}=a_{2}^{-} e^{i\left(\omega t-k_{2} x\right)}+a_{2}^{+} e^{i\left(\omega t+k_{2} x\right)}$, the expressions of $a_{2}^{-}$and $a_{2}^{+}$for a porous medium of length $L_{p}$ such as $0 \leqslant x \leqslant L_{p}$ write:

$$
\begin{aligned}
& a_{2}^{-}(x)=a_{2}^{-}(0) e^{-k_{2, d} x} \\
& a_{2}^{+}(x)=a_{2}^{+}\left(L_{p}\right) e^{-k_{2, d}\left(L_{p}-x\right)}
\end{aligned}
$$

where $k_{2, d}=k_{2} / n_{w}$ is the damping rate per unit length, $n_{w}$ is chosen constant. It is obtained empirically from comparison to experimental data (see Arnaud et al. (2017)).

\subsection{Numerical method of resolution}

We present here not only the case of porous structure but also the case of a porous structure with impervious lateral boundaries (at $y= \pm d_{p}$ ) since both are computed for comparisons with experimental data.

\subsubsection{Porous structure}

The continuity conditions for pressure $p=-\rho \frac{\partial \Phi}{\partial t}$ and velocity at vertical boundaries are applied to the vertical extremities of the porous medium:

For $x=0$ :

$$
\left\{\begin{aligned}
& \Phi_{1}=S_{r} \Phi_{2} \text { and } \frac{\partial \Phi_{1}}{\partial x}=\gamma \frac{\partial \Phi_{2}}{\partial x} \text { for } 0 \leqslant y \leqslant d_{p} \\
& \Phi_{1}=\Phi_{3} \text { and } \frac{\partial \Phi_{1}}{\partial x}=\frac{\partial \Phi_{3}}{\partial x} \quad \text { for } \quad d_{p} \leqslant y \leqslant d_{B}
\end{aligned}\right.
$$

For $x=L_{p}$ :

$$
\left\{\begin{aligned}
S_{r} \Phi_{2}=\Phi_{4} & \text { and } \gamma \frac{\partial \Phi_{2}}{\partial x}=\frac{\partial \Phi_{4}}{\partial x} \text { for } 0 \leqslant y \leqslant d_{p} \\
\Phi_{3} & =\Phi_{4} \text { and } \frac{\partial \Phi_{3}}{\partial x}=\frac{\partial \Phi_{4}}{\partial x} \text { for } d_{p} \leqslant y \leqslant d_{B}
\end{aligned}\right.
$$

For $y=d_{p}$

$$
\begin{cases}S_{r} \Phi_{2}=\Phi_{3} & \text { for } 0 \leqslant x \leqslant L_{p} \\ \gamma \frac{\partial \Phi_{2}}{\partial y}=\frac{\partial \Phi_{3}}{\partial y} & \text { for } 0 \leqslant x \leqslant L_{p}\end{cases}
$$

\section{Continuity equations between domains:}

The weak formulation of momentum and pressure continuity conditions is used by double integration over both $y$ and $z$ directions, for $n=0, \ldots, P$ based on eigenfunction expansion matching method. Integration along the $z$-axis on the water depth gives the following constants $K$ and $K_{p}$.

$$
K=\int_{-h}^{0} \cosh [k(z+h)] d z \text { and } K_{p}=\int_{-h}^{0} \cosh \left[k_{p}(z+h)\right] d z
$$

where $k=k_{i}$ for $i=1,3,4$ and $k_{p}=k_{2}$. At boundaries $x=0$ and $x=L_{p}$, classical integral matching method is used, with the choice of orthogonal eigenfunctions for 
the integral formulation. For the boundary conditions at $x=0$ and $x=L_{p}$, basic functions $\psi_{j, n}, n=0, \ldots, P$ are used ( $j=1,4$ for $x=0$ and $x=L_{p}$ respectively). For the boundary conditions at $y=d_{p}$, we consider the functions $C h_{n}(x)=\cosh \left[l_{n} x\right]$ with $l_{n}=\frac{n \pi}{L_{p}}$, which form an orthogonal set since,

$$
\int_{0}^{L_{p}} C h_{n} . C h_{m} d x=0 \text { if } n \neq m
$$

The continuity equation can then be written, for $x=0$ :

$$
\left\{\begin{aligned}
K \int_{0}^{d_{B}} \phi_{1} \psi_{1 n}(y) d y & =S_{r} K_{p} \int_{0}^{d_{p}} \phi_{2} \psi_{1 n}(y) d y+K \int_{d_{p}}^{d_{B}} \phi_{3} \psi_{1 n}(y) d y \\
K \int_{0}^{d_{B}} \frac{\partial \phi_{1}}{\partial x} \psi_{1 n}(y) d y & =\gamma K_{p} \int_{0}^{d_{p}} \frac{\partial \phi_{2}}{\partial x} \psi_{1 n}(y) d y+K \int_{d_{p}}^{d_{B}} \frac{\partial \phi_{3}}{\partial x} \psi_{1 n}(y) d y
\end{aligned}\right.
$$

for $n=0, \ldots, P$.

Similarly for $x=L_{p}$ :

$$
\left\{\begin{array}{c}
S_{r} K_{p} \int_{0}^{d_{p}} \phi_{2} \psi_{4 n}(y) d y+K \int_{d_{p}}^{d_{B}} \phi_{3} \psi_{4 n}(y) d y=K \int_{0}^{d_{B}} \phi_{4} \psi_{4 n}(y) d y \\
\gamma K_{p} \int_{0}^{d_{p}} \frac{\partial \phi_{2}}{\partial x} \psi_{4 n}(y) d y+K \int_{d_{p}}^{d_{B}} \frac{\partial \phi_{3}}{\partial x} \psi_{4 n}(y) d y=K \int_{0}^{d_{B}} \frac{\partial \phi_{4}}{\partial x} \psi_{4 n}(y) d y
\end{array}\right.
$$

$n=0, \ldots, P$ and, for $y=d_{p}$ :

$$
\left\{\begin{array}{l}
S_{r} K_{p} \int_{0}^{L_{p}} \phi_{2} C h_{n}(x) d x=K \int_{0}^{L_{p}} \phi_{3} C h_{n}(x) d x \\
\gamma K_{p} \int_{0}^{L_{p}} \frac{\partial \phi_{2}}{\partial y} C h_{n}(x) d x=K \int_{0}^{L_{p}} \frac{\partial \phi_{3}}{\partial y} C h_{n}(x) d x
\end{array}\right.
$$

$n=0, \ldots, P$.

After a development of the potential expressions (14), (16), (18) and (20), a set of $6(P+1)$ linear equations, with $6(P+1)$ unknowns $A^{ \pm}$are then to be solved.

Without dissipation by the porous medium $\left(n_{w} \rightarrow \infty\right)$, the wave energy flux along the $O x$ direction, over the basin width $d_{B}$, is conserved:

$$
\left|A_{10}^{-}\right|^{2}=\left|A_{10}^{+}\right|^{2}+\left|A_{40}^{-}\right|^{2}+\frac{1}{2} \sum_{n=1}^{n_{p}}\left[\left|A_{1 n}^{+}\right|^{2}+\left|A_{4 n}^{-}\right|^{2}\right]
$$

3.2.2. Porous structure with impervious walls at $y=d_{p}$

In order to fulfill the zero normal velocity at $y=d_{p}$,

$$
k_{2 y n}=\frac{n \pi}{d_{B}-d_{p}} \text { and } k_{2 y n}=\frac{n \pi}{d_{p}}
$$

The number of unknown coefficients is $6(P+1)$.

For $x=0$ :

$$
\left\{\begin{aligned}
K \int_{0}^{d_{p}} \phi_{1} \psi_{1 n}(y) d y & =S_{r} K_{p} \int_{0}^{d_{p}} \phi_{2} \psi_{1 n}(y) d y \\
\int_{d_{p}}^{d_{B}} \phi_{1} \psi_{1 n}(y) d y & =\int_{d_{p}}^{d_{B}} \phi_{3} \psi_{1 n}(y) d y \\
K \int_{0}^{d_{B}} \frac{\partial \phi_{1}}{\partial x} \psi_{1 n}(y) d y & =\gamma K_{p} \int_{0}^{d_{p}} \frac{\partial \phi_{2}}{\partial x} \psi_{1 n}(y) d y+K \int_{d_{p}}^{d_{B}} \frac{\partial \phi_{3}}{\partial x} \psi_{1 n}(y) d y
\end{aligned}\right.
$$


For $x=L_{p}$ :

$$
\left\{\begin{aligned}
S_{r} K_{p} \int_{0}^{d_{p}} \phi_{2} \psi_{4 n}(y) d y & =K \int_{0}^{d_{p}} \phi_{4} \psi_{4 n}(y) d y \\
\int_{d_{p}}^{d_{B}} \phi_{3} \psi_{4 n}(y) d y & =\int_{d_{p}}^{d_{B}} \phi_{4} \psi_{4 n}(y) d y \\
\gamma K_{p} \int_{0}^{d_{p}} \frac{\partial \phi_{2}}{\partial x} \psi_{4 n}(y) d y+K \int_{d_{p}}^{d_{B}} \frac{\partial \phi_{3}}{\partial x} \psi_{4 n}(y) d y & =K \int_{0}^{d_{B}} \frac{\partial \phi_{4}}{\partial x} \psi_{4 n}(y) d y
\end{aligned}\right.
$$

Without dissipation, condition (31) is still verified.

\section{Results and discussions}

Wave amplitude fields around the porous medium have been measured for the wave conditions given in Table 1. Our first aim was to analyse the wave scattering by the porous structure including reflection, refraction-diffraction and dissipation phenomena. Let us note that the side walls of the basin may affect the wave behaviour near the structure, since $d_{B}$ is not large compared to $d_{p}$. Such a configuration can also describe the case of a periodic detached breakwater system (Rey et al., 2018). Intermediate water depth conditions are considered for $f=1$ and $1.5 \mathrm{~Hz}$, while $f=1.8$ $\mathrm{Hz}$ nearly corresponds to deep water conditions since $h / \lambda=0.48$ and $h / \lambda_{p}=0.53$ where $\lambda_{p}=2 \pi / k_{p}$. For the three wave conditions, the wave's wavelength remains much higher than the cylinders size and spacing. The length of the porous structure remains lower than the wavelength for the three frequency cases, while the width $2 d_{p}=1.20 \mathrm{~m}$ is of the order of the wavelength for $f=1 \mathrm{~Hz}$, twice the wavelength for $f=1.5 \mathrm{~Hz}$ and five times for $f=1.8 \mathrm{~Hz}$.

\subsection{Maps of wave amplitude}

Figures 4 to 6 depict 2D maps of relative wave amplitudes for each wave frequency, for incoming wave amplitude $0.010 \mathrm{~m}$ for $f=1 \mathrm{~Hz}$ and $0.015 \mathrm{~m}$ for $f=1.5 \mathrm{~Hz}$ and $f=$ $1.8 \mathrm{~Hz}$. Results concerning the other amplitudes (see table 1) are shown and discussed in sections 4.2 and 4.3 for longitudinal and transversal axes, respectively. Relative wave amplitude corresponds to the ratio between the wave amplitude measured around the porous medium and the wave amplitude measured at the position of the reference wave gauge upwave the structure (see Fig. 1). For each case, plots of wave amplitude from the experimental results are compared to numerical results for the porous structure. The experimental wave fields are obtained after using a cubic spline interpolation technique.

For the first studied frequency $f=1 \mathrm{~Hz}$ and $a=0.010 \mathrm{~m}$, the wave amplitude response upstream the porous structure along the central axis of the basin presents successive maxima and minima, representing a wave reflection phenomenon (Fig. 4, top). Downstream the porous structure the wave amplitude is rather homogeneous; a part of the wave being transmitted through the porous structure. Wave refractiondiffraction is observed on both edges of the structure.

Figure 4 (bottom) represents the theoretical model with a porous structure. The wave reflection is well represented regarding the $3 \mathrm{D}$ wave amplitude pattern. 
The wave amplitude field downstream of the porous structure appears to be smooth do the transmission of a significant part of the wave energy through the structure. We can notice however that the amplitude variations are more pronounced for the experimental case.

For $f=1.8 \mathrm{~Hz}$, the experimental result (Fig. 6, top) shows wave reflection as for $f=1 \mathrm{~Hz}$ but the extrema of wave amplitude upstream the porous structure are more numerous due to a higher wavelength. Downstream the structure the wave rays are curved particularly near to structure sides. The transmission is also lower than for the first studied frequency. The theoretical model (Fig. 6, bottom) fits correctly the experimental results. The oscillations of wave amplitude upstream the structure are well represented as the wave rotation downstream the porous medium.

For the wave frequency $f=1.5 \mathrm{~Hz}$, the experimental data give another pattern compared to other cases. The wave amplitude oscillations is still observed along the wave propagation direction but another amplitude oscillation is observed along the transverse direction. Downstream of the structure, such a wave behaviour is also observed even if it is less pronounced due to wave dissipation and backscattering. Such transverse oscillations are well predicted qualitatively by both theoretical models for the porous medium (Fig. 5, bottom).

To summarize these results, the first overall observation is the presence of significant wave reflection and refraction-diffraction by the porous medium together with a partial transmission which smooths the diffraction pattern downwave the structure. Upstream the structure $(y<0)$, the series of successive minima and maxima can be seen as the result of the partial reflection by the porous medium even if diffraction effects arising from the structure edges may modify the wave patterns. These spatial modulations of wave amplitude upstream the porous block are observed for the three wave conditions, with an increasing number of oscillations for increasing wave frequency. Downstream of the porous medium, refraction-diffraction effects can be observed. The wave direction varies along the basin width, propagating faster on both sides of the structure and forming a cylindrical-type wave. The wave energy downstream the porous medium results from the combination of transmission through the porous medium and diffraction at its edges, producing a more complex wave structure than for an impervious structure. However, some discrepancies are observed between experimental data and theoretical results. They are certainly due to wave scattering from the beach and from the basin walls. Indeed, the basin and the beach are designed for wave propagation along the $\mathrm{x}$-axis, scattered waves by the porous structure may be reflected by the beach, not designed for oblique incidence.

The theoretical results for the porous structure are found to provide a correct representation of the surface wave field obtained for the three studied frequencies. Since a given dissipation rate $n_{w}$ (Arnaud et al., 2017) per unit wavelength is considered, higher dissipation is observed for the higher frequency case, the porous length $L_{p}$ being kept constant in the experiments. For $f=1.5 \mathrm{~Hz}$, we can also observe max- 
March

18 ,

2019

$14: 39$

FILE
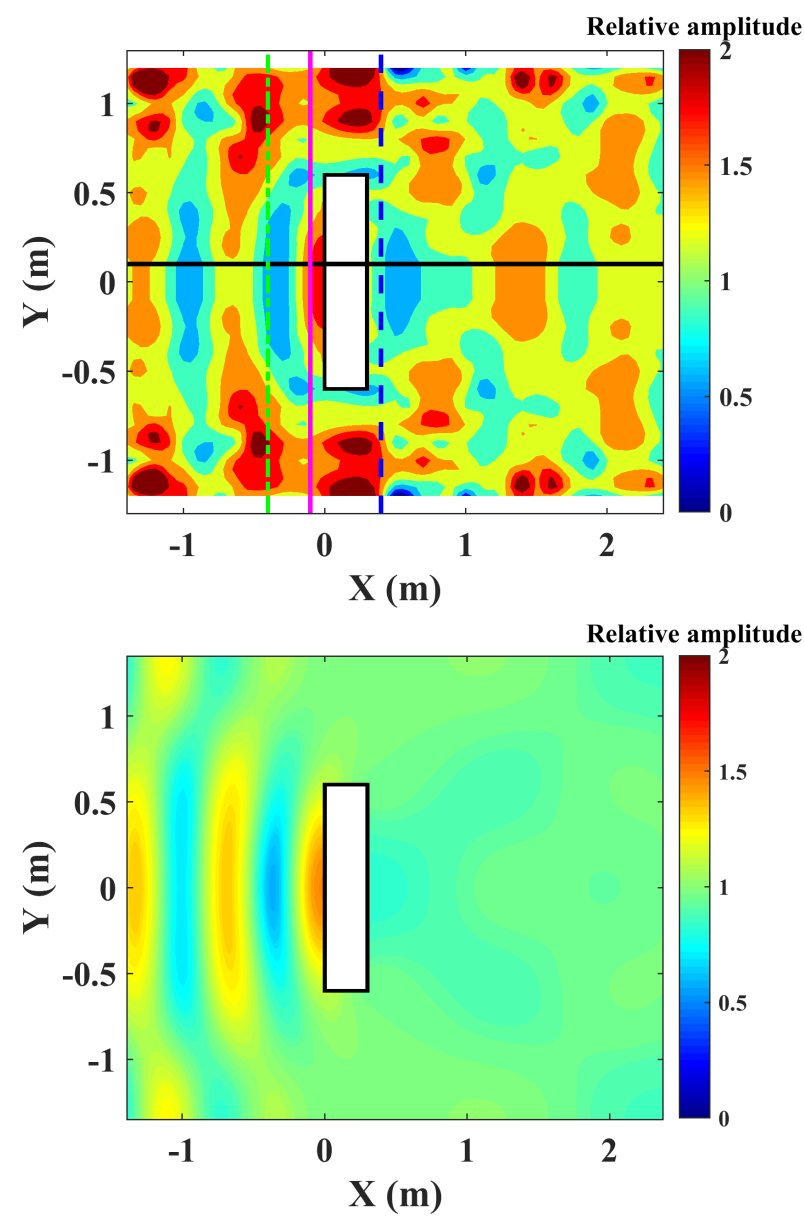

Figure 4. Wave amplitude for $f=1 \mathrm{~Hz}$ experiments (top), theory (down)

ima and minima of amplitude along the cross section ascribed to resonance effects since the gap between the side walls and the porous medium $(0.7 \mathrm{~m})$ corresponds nearly to the wave wavelength $(\lambda=0.68 \mathrm{~m})$ and the porous width $\left(2 d_{p}=1.20 \mathrm{~m}\right)$ to the wave wavelength inside the porous medium $\left(\lambda_{p}=0.61 \mathrm{~m}\right)$.

\subsection{Wave amplitude along a longitudinal transect}

Measurements along the section $y=0.10 \mathrm{~m}$, i.e. near the longitudinal axis of the basin, are compared to the 3D model results for the porous structure. The results are also compared to the 2D theoretical model neglecting structure edges lateral effects $\left(d_{p} \rightarrow \infty\right)$ (Arnaud et al., 2017).

Overall, Figure 7 shows that the theoretical results for the 3D case for a porous structure are in good agreement with experimental data both upstream and down- 
March

18,

$2019 \quad 14: 39$
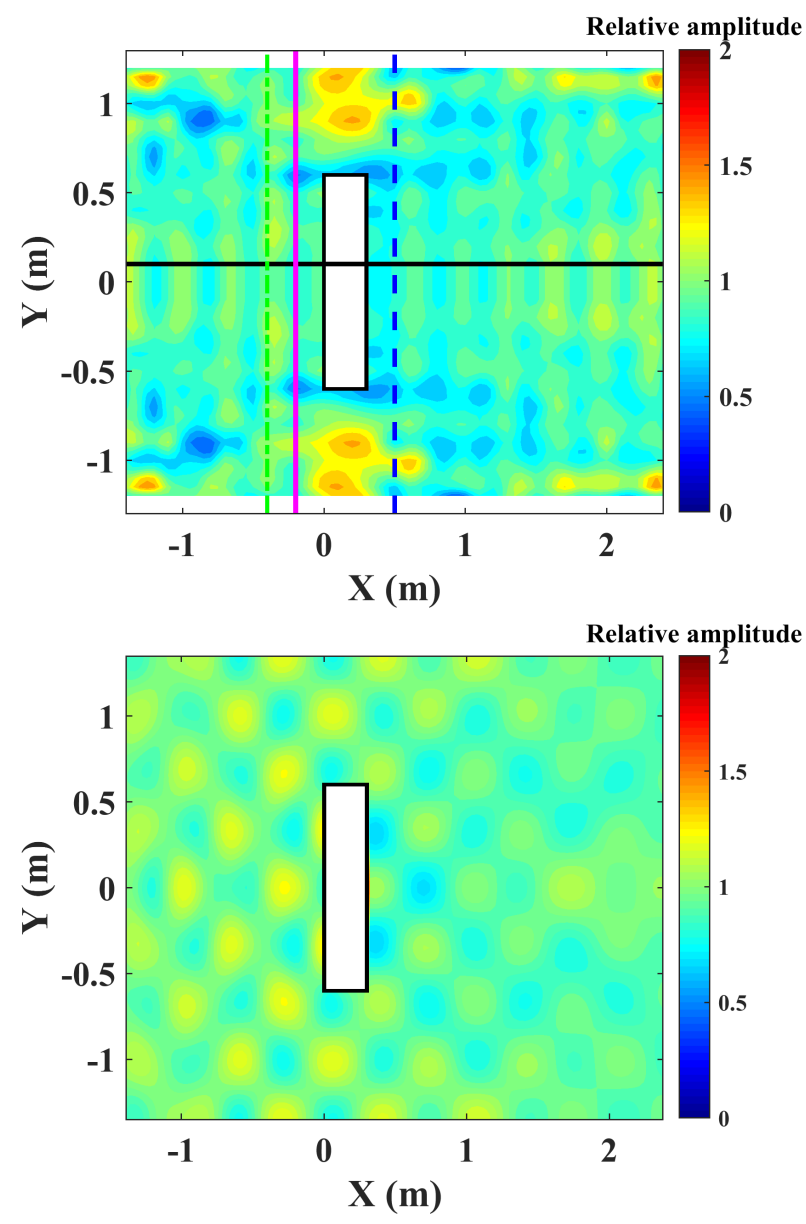

Figure 5. Wave amplitude for $f=1.5 \mathrm{~Hz}$ experiments (top), theory (down)

stream the structure. The dissipation as well as reflection and refraction-diffraction are well represented by the model. The amplitude study shows that the results are consistent with the linear assumptions considered in the model. Indeed, quasi-similar amplitudes are observed whatever the incoming wave amplitude as shown both on longitudinal and transversal transects.

Upstream the structure, the amplitude of wave modulation is greater when increasing frequency. This is induced by the frequency dependency of the reflected wave amplitude due to interference processes shown in Arnaud et al. (2017). Downstream the structure, the sheltering effect of the porous medium progressively decreases and the wave amplitude increases slowly with some weak oscillations.

Frequency effects are highlighted when comparing porous 2D and 3D model. For frequencies $f=1 \mathrm{~Hz}$ (Fig. 7, top) and $f=1.8 \mathrm{~Hz}$ (Fig. 7, bottom) both models 
March

18,

2019

$14: 39$

WSPC/INSTRUCTION

FILE

Arnaud-et-al'IJOCE'Review

Wave propagation through dense vertical cylinder arrays: 3D experimental study
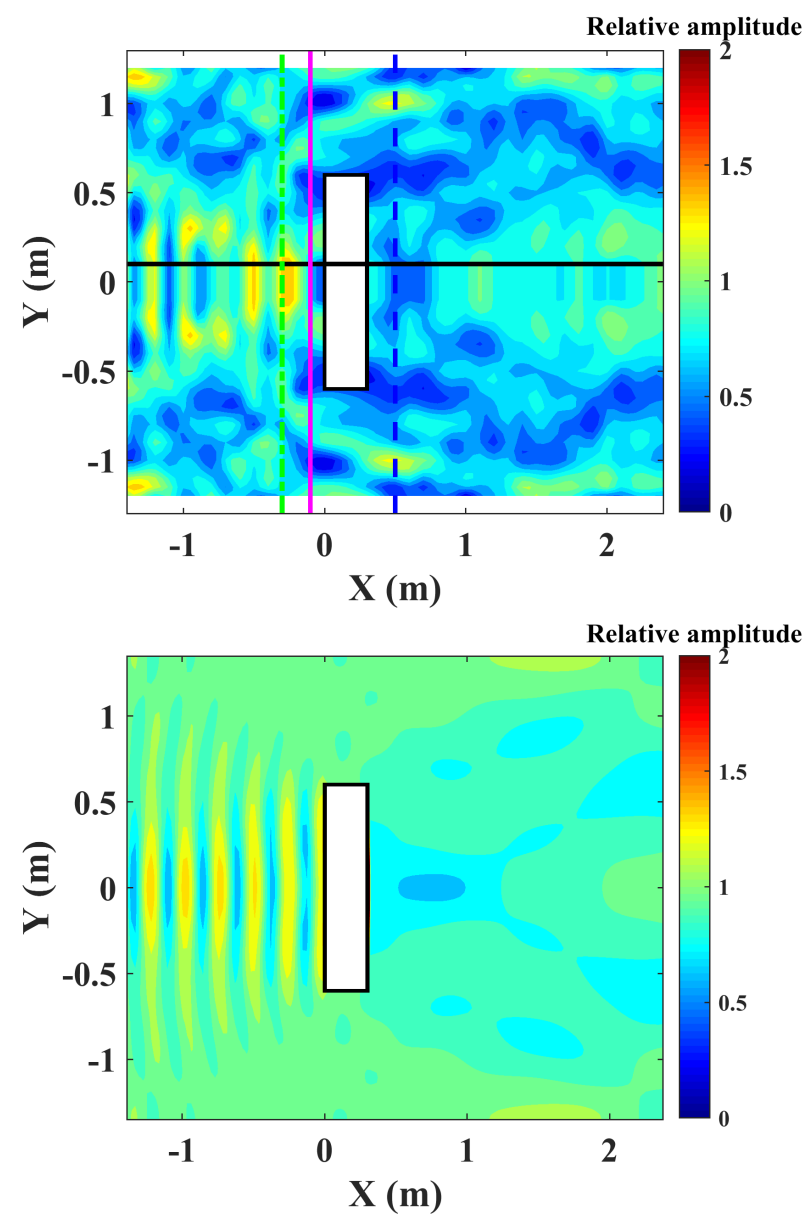

Figure 6. Wave amplitude for $f=1.8 \mathrm{~Hz}$ experiments (top), theory (down)

upstream the structure are very close. This indicates that side effects are weak along the median axis. By constrast, the case $f=1.5 \mathrm{~Hz}$ (Fig. 7, middle) highlights the influence of the finite width of the structure with significant differences between $3 \mathrm{D}$ and $2 \mathrm{D}$ cases. This discrepancy can be explained by the presence of transverse resonance in the $3 \mathrm{D}$ case. This resonant mode may then be due to either or both the basin width or the gap between the basin and side walls of the porous structure.

In addition, one note also that downstream the structure for $f=1 \mathrm{~Hz}$ large amplitude oscillations are measured but not provided by the models. They may be due to resonance behaviour between the structure and the absorbing beach used in the basin which is not perfect at low frequencies. The theoretical result with a wave reflection of the beach of $20 \%$ highlights the presence of an amplitude modulation downstream according to the observed modulation. However the predicted oscillation remains much lower even if beach reflection is taken into account in the model (Fig. 
7, top). A possible explanation for this for this discrepancy might be related to a $y$ dependent reflection of the beach, resulting from wave focusing behind the structure.

\subsection{Wave amplitude along a transversal transect}

The comparison between measurements and numerical results along the transversal direction of the basin around the porous structure is presented in Fig. 8.

These plots highlight the 3D effects of the porous structure on the wave propagation. The position of the transects are chosen to corresponds to minimum and maximum upwave the cylinder array, respectively. The general tendency of the wave amplitude is well represented by the model, the minima and maxima are well positioned along the basin width although the experimental results show higher amplitude for $f=1 \mathrm{~Hz}$ and smaller amplitude for $f=1.8 \mathrm{~Hz}$.

\subsection{Discussion on the wave resonance}

In order to better understand the observed transverse resonance occurring for $f=$ $1.5 \mathrm{~Hz}$, we present here results from a 3D model configuration neglecting the fluxes through the side walls of the porous medium. A comparison of measured wave amplitude and both theoretical cases (porous and impervious lateral boundaries) for the three studied frequencies is depicted in Fig. 9.

For frequency $f=1 \mathrm{~Hz}$ (Fig. 9, top), the wave amplitude modulations are similar for both theoretical models. This indicates that reflection and transmission are not influenced by the exchanges through the side walls of the porous structure. For $f=1.5 \mathrm{~Hz}$ (Fig. 9, middle), significant discrepancies are observed between model configurations with strong differences of wave modulations along the incoming wave direction, upstream and downstream the porous structure. As already mentioned in the previous section, the model which considers porous side walls suits better with experimental data, particularly upstream of the structure with a good representation of the extrema. This indicates that fluxes and/or pressure drops across the porous side walls have a significant influence at this frequency due to transverse resonance, while they are much less significant for $f=1 \mathrm{~Hz}$ and $f=1.8 \mathrm{~Hz}$.

For the higher frequency, $f=1.8 \mathrm{~Hz}$ (Fig. 9, bottom) both models give same results upstream of porous medium and are in good agreement with experimental data. This means that upstream the center part of porous structure there is no 3D effect as for $f=1 \mathrm{~Hz}$. Downwave the structure, the modulation is found to be higher when the structure sides are considered as porous walls. Both theoretical trends fit with experimental data even if oscillations observed experimentally may be due to beach scattering as already mentioned in the previous subsection (subsec. 4.2). The diffraction effects are then significant downstream also in its central part because the wave wavelength is of the same order as the porous length.

In the absence of the transverse resonance, wave behaviour upstream the struc- 
ture far from its edges can be well described by the 2D model. By contrast, in the presence of transverse resonance, the whole wave field is affected by $3 \mathrm{D}$ effects.

\section{Conclusions}

This combined experimental and theoretical study was dedicated to the analysis of the interactions between regular waves and porous structure of finite dimensions.

Measurements of the wave field in the presence of the porous structure allowed to evidence the effect of a porous structure on the wave scattering. Further insight was provided by the theoretical approaches, which compare 2D and 3D models. The 3D model provides a satisfactory representation of the wave field around the structure, confirming the validity of the approach. The discrepancies observed between model and measurements downstream the structure especially at the lower frequency are attributed to wave scattering by the absorbing beach which hardly dissipates wave energy at low frequency in the experiments.

Transverse resonances related to both structure width and the basin side walls are shown by the measurements and accurately modelled. Two configurations, with porous and impervious lateral boundaries, are used in the model to better understand such processes. The resonance effect by the porous structure is amplified when side-walls are considered as impervious. The difference between both hypothesis in theoretical models is significant only for the case $f=1.5 \mathrm{~Hz}$ due to the presence of transverse resonance, whereas the difference is rather negligible for both other studied wave conditions.

On the whole, model gives a good prediction of wave transformation processes through and around porous structure of finite dimensions. Further efforts will be engaged to extend the model to more complex configurations of porous medium, in particular including inhomogeneous porosity or specific surface within the structure.

\section{Acknowledgements}

The authors would like to thank the Region Provence Alpes Côte d'azur and the company ACRI-IN for their financial contributions of the PhD thesis scholarship of Gwendoline Arnaud that allowed this work. The authors are grateful Aimed Ajroud for his help to the physical models conception.

\section{References}

Abul-Azm, A. G. and Williams, A. N. (1997). Oblique wave diffraction by segmented offshore breakwaters. Ocean Engineering, 24(1):63-82.

Arnaud, G., Rey, V., Touboul, J., Sous, D., Molin, B., and Gouaud, F. (2017). Wave propagation through dense vertical cylinder arrays : Interference process and specific surface effects on damping. Applied Ocean Research, 65:229-237.

Chang, C.-w., Liu, P. L., Mei, C. C., and Maza, M. (2017a). Modeling transient 
long waves propagating through a heterogeneous coastal forest of arbitrary shape. Coastal Engineering, 122(January):124-140.

Chang, C.-w., Liu, P. L., Mei, C. C., and Maza, M. (2017b). Periodic water waves through a heterogeneous coastal forest of arbitrary shape. Coastal Engineering, 122(January):141-157.

Dalrymple, R. A. and Martin, P. A. (1990). Wave diffraction through offshore breakwaters. Journal of waterway, port, coastal, and ocean engineering, 116(6):727-741.

Kagemoto, H. and Yue, D. K. P. (1986). Interactions among multiple threedimensional bodies in water waves: an exact algebraic method. Journal of Fluid Mechanics, 166:189-209.

Liu, P. L. F., Chang, C. W., Mei, C. C., Lomonaco, P., Martin, F. L., and Maza, M. (2015). Periodic water waves through an aquatic forest. Coastal Engineering, 96:100-117.

Lowe, R. J. (2005a). Oscillatory flow through submerged canopies: 1. Velocity structure. Journal of Geophysical Research: Oceans (1978-2012), 110(C10).

Lowe, R. J. (2005b). Oscillatory flow through submerged canopies: 1. Velocity structure. Journal of Geophysical Research: Oceans (1978-2012), 110(C10).

Ma, G., Kirby, J. T., Su, S. F., Figlus, J., and Shi, F. (2013). Numerical study of turbulence and wave damping induced by vegetation canopies. Coastal Engineering, 80:68-78.

Maza, M., Lara, J. L., and Losada, I. J. (2015). Tsunami wave interaction with mangrove forests: a 3-D numerical approach. Coastal Engineering, 98:33-54.

Mei, C. C., Chan, I. C., and Liu, P. L. F. (2014). Waves of intermediate length through an array of vertical cylinders. Environmental Fluid Mechanics, 14:235261.

Ohl, C., Eatock Taylor, R., Taylor, P., and Borthwick, A. (2001). Water wave diffraction by a cylinder array. Part 1. Regular waves. Journal of Fluid Mechanics, 442:1-32.

Rey, V., Arnaud, G., Touboul, J., and Belibassakis, K. (2018). Water wave scattering by dense or sparse arrays of surface-piercing bodies by integral matching method. Applied Ocean Research, 75:132-142.

Sollitt, C. and Cross, R. (1972). Wave transmission through permeable breakwaters. Coastal Engineering Proceedings, 1(13):1827-1846.

Yang, Y., Irish, J. L., and Socolofsky, S. A. (2015). Numerical investigation of wave-induced fl ow in mound - channel wetland systems. Coastal Engineering, 102:1-12.

Yu, X. and Chwang, A. T. (1994). Wave motion through porous structures. Journal of Engineering Mechanics, 120(5):989-1008. 
March

18

2019

14:39

FILE

Arnaud-et-al'IJOCE'Review
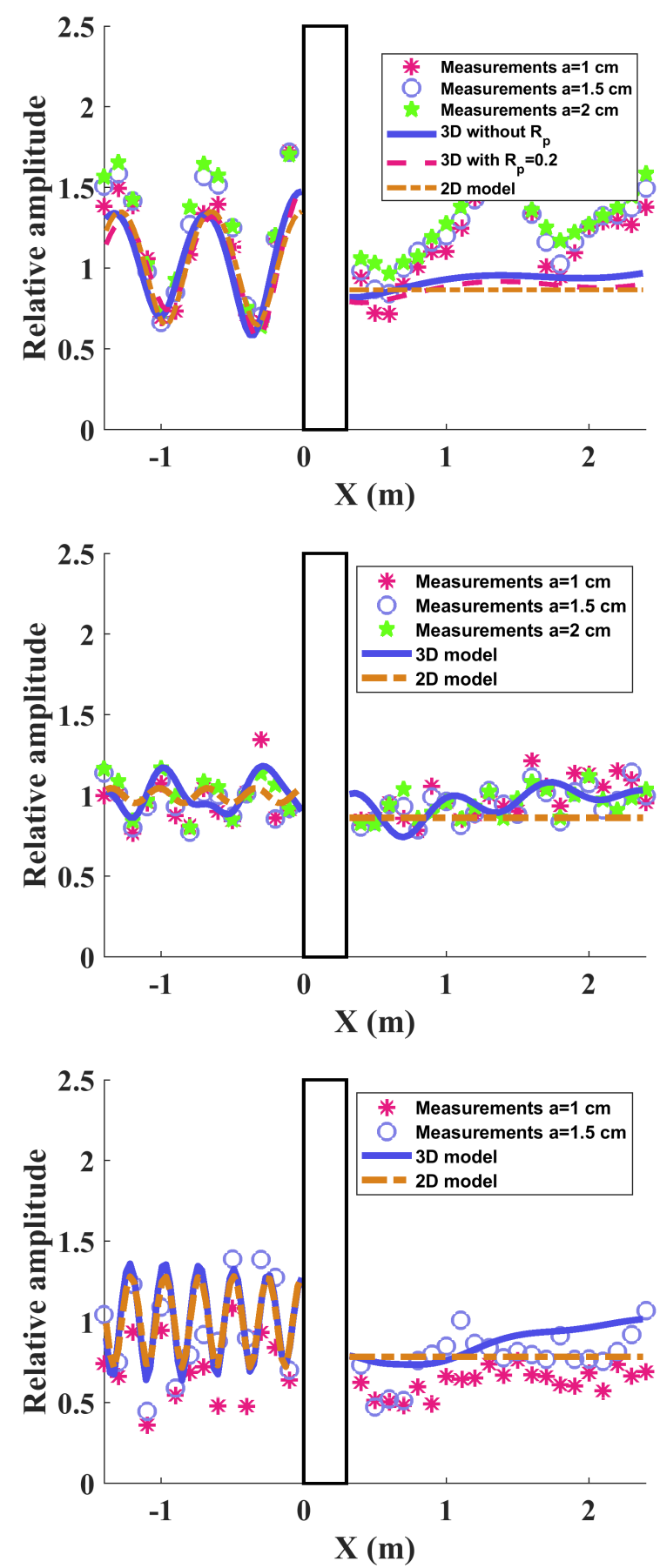

Figure 7. Cut near to the axis of the basin $(Y=0.10 \mathrm{~m}$ ) for $f=1 \mathrm{~Hz}$ (top), $f=1.5 \mathrm{~Hz}$ (middle) and $f=1.8 \mathrm{~Hz}($ down $)$ 

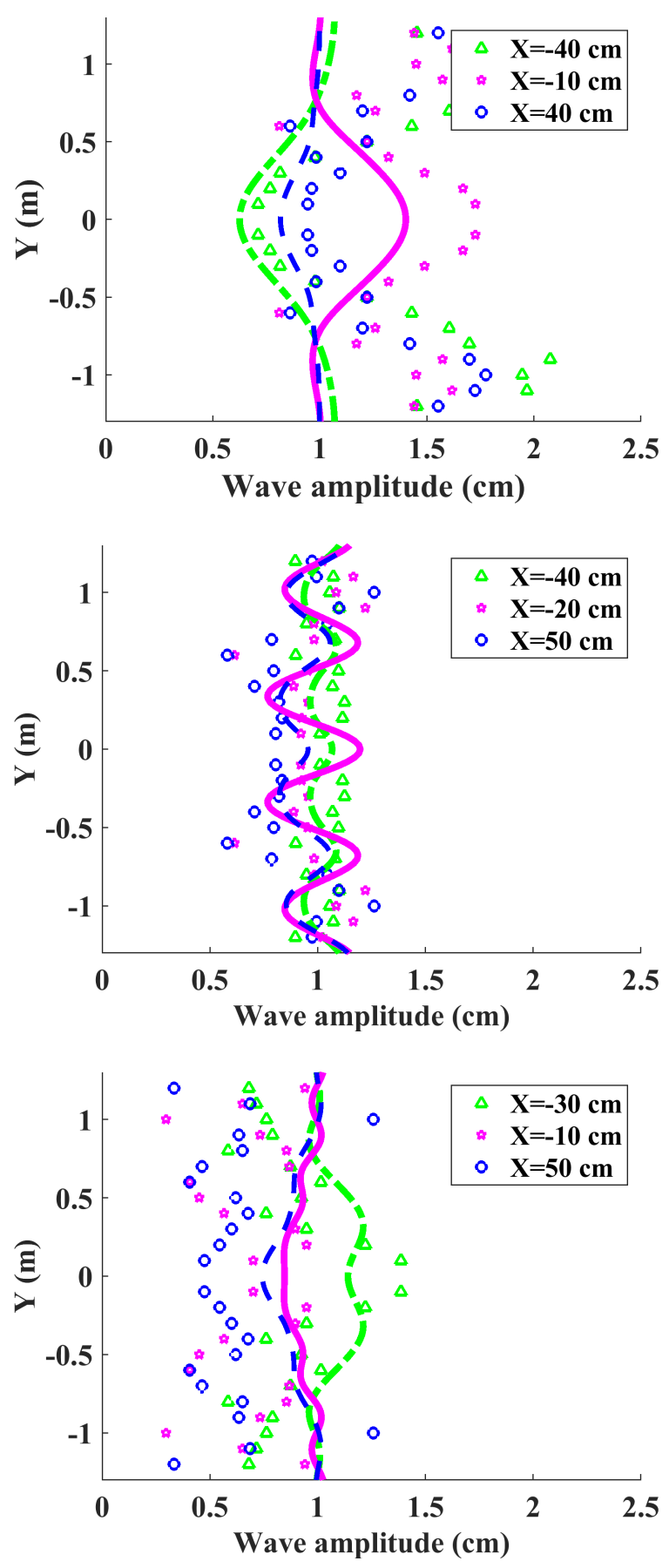

Figure 8. Transversal cuts of the basin for $f=1 \mathrm{~Hz}$ (top), $f=1.5 \mathrm{~Hz}$ (middle) and $f=1.8 \mathrm{~Hz}$ (down) 
March

18 ,

Arnaud-et-al'IJOCE'Review
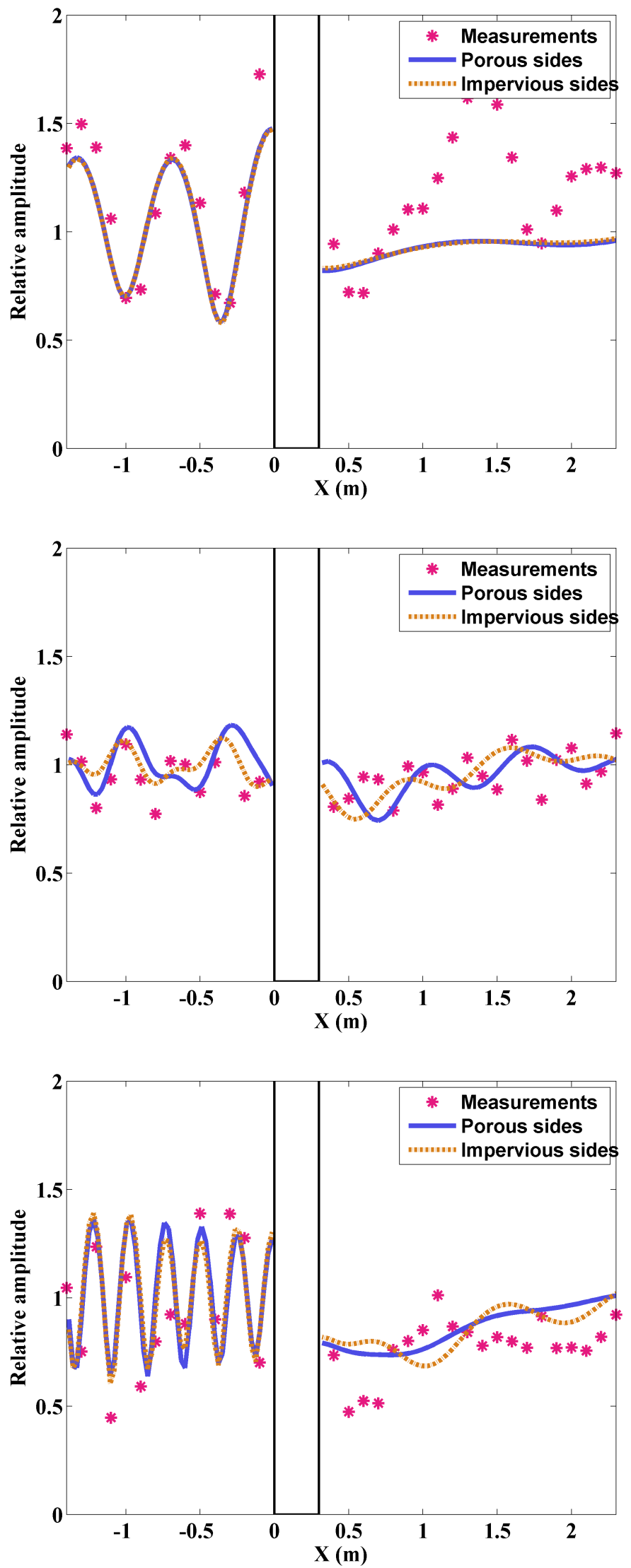

Figure 9. Cut near to the axis of the basin $(Y=0.10 \mathrm{~m}$ ) for $f=1 \mathrm{~Hz}$ (top), $f=1.5 \mathrm{~Hz}$ (middle) and $f=1.8 \mathrm{~Hz}$ (down) 